Check for updates

Cite this: Chem. Commun., 2021,

57, 2376

Received 15th December 2020,

Accepted 25th January 2021

DOI: $10.1039 / \mathrm{d} 0 \mathrm{cc} 08144 \mathrm{~b}$

rsc.li/chemcomm

\section{Hypersensitive pressure-dependence of the conversion temperature of hysteretic valence tautomeric manganese-nitronyl nitroxide radical 2D-frameworks $\dagger$}

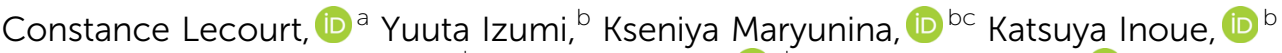 \\ Nicolas Bélanger-Desmarais, ${ }^{d}$ Christian Reber, (D) ${ }^{d}$ Cédric Desroches $\mathbb{D D}^{a}$ and \\ Dominique Luneau (D) *a
}

\begin{abstract}
Valence tautomeric manganese(II)-radical lamellar compounds $\left\{\left[\mathrm{Mn}_{2}(\mathrm{NITIm})_{3}\right] \mathrm{X}\right\}_{\mathrm{n}}$ with NITIm a nitronyl nitroxide radical and $\mathrm{X}=\mathrm{ClO}_{4}{ }^{-}$(1) or $\mathrm{BF}_{4}{ }^{-}$(2) show a pressure-induced increase of their conversion temperature by approximately $40 \mathrm{~K}$ at a mild external pressure of $0.1 \mathrm{GPa}$, shifting the transition from near room temperature to hot temperature regions.
\end{abstract}

Multistable magnetic coordination compounds that are switchable by external stimuli such as heat, pressure or light deserve to be investigated. ${ }^{1}$ They are indeed useful to gain better, broad-based insight on fundamental phenomena such as intramolecular electron transfer processes and their relationships with particular solidstate rearrangements. ${ }^{2}$ Moreover, these compounds are of interest for technological applications as switches, sensors or in signal processing and information storage devices. ${ }^{3}$ New examples among these magnetic molecular switches are valence tautomeric (VT) coordination compounds with intramolecular electron transfers between metal ions and redox-active ligands that cause switching between different redox tautomer forms. ${ }^{4}$ Valence tautomerism is an alternative to spin-crossover (SCO), with which it shares several similarities. ${ }^{4-7}$ Most reports on VT compounds deal with conversions triggered by temperature variations, with a smaller number of studies on the effect of pressure ${ }^{8-12}$ or electric field variations. ${ }^{13}$ Most VT compounds are based on dioxolene derivatives as redoxactive ligands ${ }^{4,7,14}$ and only a few examples use free radicals such as

\footnotetext{
${ }^{a}$ Univ Lyon, Université Claude Bernard Lyon 1, CNRS, Laboratoire des Multimatériaux et Interfaces, F-69622 Villeurbanne, France. E-mail: dominique.luneau@univ-lyon1.fr

${ }^{b}$ Department of Chemistry, Graduate School of Science and Chirality Research Center (CResCent), Hiroshima University, 1-3-1, Kagamiyama, Higashi Hiroshima, Hiroshima, 739-8526, Japan

International Tomography Center SB RAS, 3A Institutskaya Street, Novosibirsk 630090, Russia

${ }^{d}$ Département de Chimie, Université de Montréal, Montréal, Québec, H3C 3J7, Canada

$\dagger$ Electronic supplementary information (ESI) available. See DOI: 10.1039/ d0cc08144b
}

verdazyl $^{15}$ or nitronyl nitroxide, as in manganese coordination compounds we recently documented. ${ }^{16,17}$ The latter are lamellar crystalline compounds made of $2 \mathrm{D}$ metal-radical coordination polymers formulated as $\left\{\left[\mathrm{Mn}_{2-y}^{\text {II }} \mathbf{M n}_{y}^{\text {III }}(\mathrm{NITIm})_{3-y}(\mathrm{NITRed})_{y}\right]^{+}\right\}_{n}$ separated by layers of counter-anions (X). Cooling down the compounds leads to oxidation of $\mathrm{Mn}$ (II) to $\mathrm{Mn}$ (III) and reduction of nitronyl nitroxide radicals $\left(\mathrm{NITIm}^{-}\right)$to their aminoxyl form $\left(\mathrm{NITRed}_{2}{ }^{-}\right)$ (Scheme 1 and Scheme S1, ESI $\dagger$ ). The electron transfers reverse upon reheating with hysteresis. The compound with $\mathrm{X}=\mathrm{ClO}_{4}{ }^{-}(\mathbf{1})^{16}$ was first discovered and the family was recently expanded to compounds with $\mathrm{X}=\mathrm{BF}_{4}{ }^{-}$(2) or $\mathrm{PF}_{6}{ }^{-}(3){ }^{17}$

The three compounds are isostructural and differ only by their anions. They are an intriguing example of chemical modulation. ${ }^{1}$ The conversion occurs in one step for $\mathbf{1}$, from the high temperature form (HT, $\left\{\mathbf{M n}_{\mathbf{2}}^{\text {II }}\right\}, y=0$ ) to the low temperature form (LT, $\left.\left\{\mathbf{M n}_{\mathbf{2}}^{\text {III }}\right\}, y=2\right)\left(\mathbf{1}_{\mathbf{H T}} \Leftrightarrow \mathbf{1}_{\mathbf{L T}}\right)$, and in two steps for $\mathbf{2}$ and $\mathbf{3}$, from HT to LT though an intermediate form (INT, $\left.\left\{\mathbf{M n}^{\mathrm{II}} \mathbf{M n}{ }^{\mathrm{III}}\right\}, y=1\right)\left(\mathbf{2}_{\mathbf{H T}} \Leftrightarrow \mathbf{2}_{\mathrm{INT}} \Leftrightarrow \mathbf{2}_{\mathbf{L T}}\right.$ and $\mathbf{3}_{\mathbf{H T}} \Leftrightarrow$ $\mathbf{3}_{\text {INT }} \Leftrightarrow \mathbf{3}_{\text {LT }}$ ). Compounds $\mathbf{1}$ and 2 show broad and abrupt hysteresis whereas it is narrow and gradual for compound 3. ${ }^{16,17}$ At room temperature, 1 and 2 are in their HT form $\left(\mathbf{1}_{\mathbf{H T}}\right.$ and $\left.\mathbf{2}_{\mathbf{H T}}\right)$ and $\mathbf{3}$ in its LT form $\left(\mathbf{3}_{\mathbf{L T}}\right)$. This is ascribed to the bigger size of the $\mathrm{PF}_{6}{ }^{-}$anion which should induce a higher

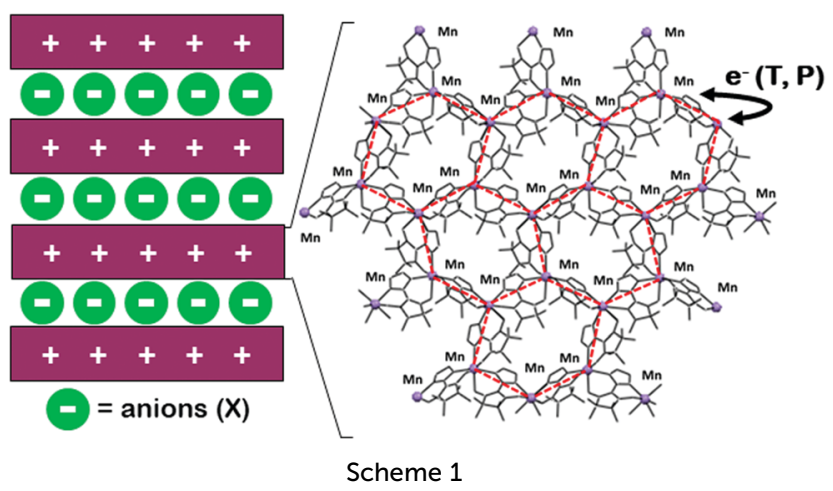




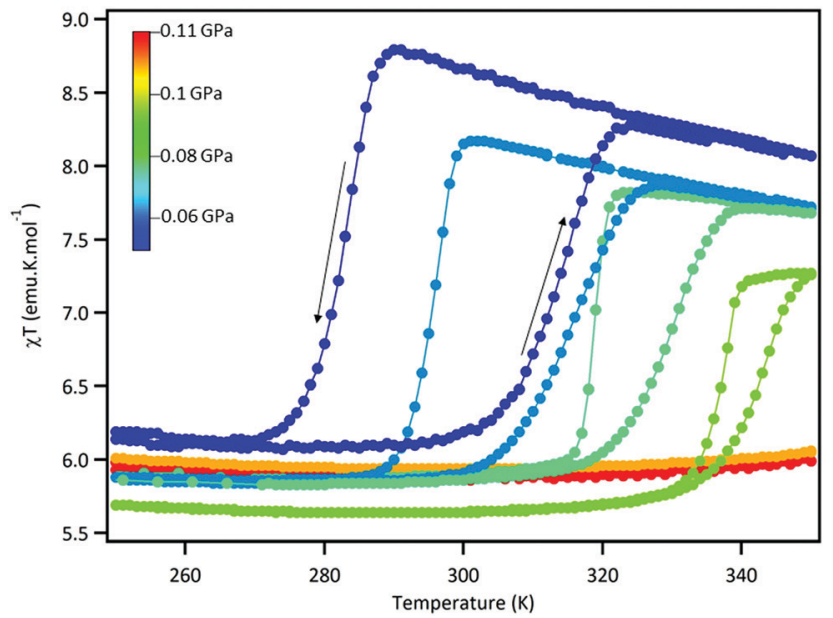

Fig. $1 \chi T$ vs. $T$ curves of compound 1 in the temperature range $250-350 \mathrm{~K}$ and at variable pressure ranging in between $10^{-4} \mathrm{GPa}$ (dark blue) and $0.11 \mathrm{GPa}$ (red). Black arrows indicate the direction of the hysteresis and solid lines only join the data points.

chemical pressure than the smaller $\mathrm{ClO}_{4}{ }^{-}$and $\mathrm{BF}_{4}{ }^{-}$, and therefore stabilizes the $\mathbf{3}_{\mathbf{L S}}$ form of smaller volume as observed for SCO systems. ${ }^{18}$ This phenomenon motivated us to study the effect of pressure on these systems.

We report here the magnetic study and Raman spectroscopy of compounds $\mathbf{1}$ and $\mathbf{2}$ under pressure. This was not performed for compound 3 because the temperature range of its VT conversion (330-380 K) falls outside the permitted temperature limit $(350 \mathrm{~K})$ of the Daphne 7373 pressure-transmitting medium. ${ }^{19}$

At atmospheric pressure, compound $\mathbf{1}$ shows a very abrupt conversion with broad hysteresis. ${ }^{16}$ The temperatures at halfconversion are $277 \mathrm{~K}$ for $T_{1 / 2} \downarrow$ and $296 \mathrm{~K}$ for $T_{1 / 2} \uparrow$ and the hysteresis width $(\Delta T)$ is $19 \mathrm{~K}$.

Fig. 1 shows the $\chi T$ vs. $T$ measurements of compound 1 at variable pressure $\left(10^{-4}-0.11 \mathrm{GPa}\right)$ in the temperature range from 250 to $350 \mathrm{~K}$. At $10^{-4} \mathrm{GPa}$, when the sample is just confined in the oil used for transmitting hydrostatic pressure, the conversion temperature increases $\left(T_{1 / 2} \downarrow=283 \mathrm{~K}\right.$ and $\left.T_{1 / 2} \uparrow=313 \mathrm{~K}\right)$ and the hysteresis width broadens $(\Delta T=31 \mathrm{~K})$. This behavior is occasionally observed for compounds immersed in oil as a pressure-transmitting medium..$^{20,21}$ It is ascribed to liquid oil medium/matrix effect toward the dispersed powder as may be also found for nanoparticle of $\mathrm{SCO}^{22}$ Here this illustrates the exceptionally high pressure sensitivity of compound $\mathbf{1}$. Upon pressure increase, the VT conversion shifts to higher temperatures and the hysteresis narrows while the $\chi T$ value at $350 \mathrm{~K}$ is steeply decreasing. The cooling branches remain steep compared to the heating ones which become more gradual, leading to asymmetric hysteresis. For $P>0.11 \mathrm{GPa}$, the conversion between the low temperature form and the high temperature form $\left(\mathbf{1}_{\mathbf{L T}} \rightarrow \mathbf{1}_{\mathbf{H T}}\right)$ is no longer visible within the permitted temperature range and only the $\mathbf{1}_{\mathbf{L T}}$ redox form is present. This means that at high pressure when the high temperature limit is reached, almost none of the $\left\{\mathbf{M n}_{\mathbf{2}}^{\text {III }}\right\}$ redox tautomer form reduced back to $\left\{\mathbf{M n}_{\mathbf{2}}^{\text {II }}\right\}$. Nevertheless, after a full decompression, the initial magnetic behavior is recovered (Fig. S1, ESI $\dagger$ ).
At atmospheric pressure, compound 2 exhibits a two-step valence tautomeric conversion close to room temperature with temperatures $T_{1 / 2} \downarrow$ and $T_{1 / 2} \uparrow$ of $276 \mathrm{~K}$ and $280 \mathrm{~K}$ for the first step and 236 and $253 \mathrm{~K}$ for the second step, leading to hysteresis widths of 4 and $17 \mathrm{~K}$, respectively. ${ }^{17}$

Fig. 2 shows the $\chi T$ vs. $T$ curves of compound 2 in the temperature range from 200 to $350 \mathrm{~K}$ when increasing the pressure $\left(10^{-4}-0.12 \mathrm{GPa}\right)$. Comparison with magnetic measurements under ambient-pressure, ${ }^{17}$ shows that here again the simple confinement of the sample in oil medium at $10^{-4} \mathrm{GPa}$ leads to a widening of the hysteresis $(\Delta T=6$ and $32 \mathrm{~K})$ and an increase of the temperatures at half-conversion $\left(T_{1 / 2} \downarrow=294\right.$ and $250 \mathrm{~K}, T_{1 / 2} \uparrow=280$ and $\left.300 \mathrm{~K}\right)$. The VT conversion is observed at higher temperatures with increasing pressure up to $0.12 \mathrm{GPa}$. The first hysteresis $\left(\mathbf{2}_{\mathbf{H T}} \Leftrightarrow \mathbf{2}_{\mathbf{I N T}}\right)$ has a width of $1 \mathrm{~K}$ under a pressure close to $0.07 \mathrm{GPa}$ and it disappears completely at higher pressure. Moreover, the conversion during this first step is getting more and more gradual. The second hysteresis $\left(2_{\text {INT }} \Leftrightarrow 2_{\text {LT }}\right.$ ) narrows continuously with increasing pressure and seems to vanish for $P>0.11 \mathrm{GPa}$. The conversion remains abrupt even if the warming branch becomes asymmetrical compared to the cooling one, as observed for compound $\mathbf{1}$. $\chi T$ values at $350 \mathrm{~K}$ are decreasing with increasing pressure. At the highest pressures, the VT conversion is not completed when the experimental temperature limit is reached. Nevertheless, the $\chi T$ vs. $T$ curves still show a positive slope at this temperature ascribe to a slight increase in the $\left\{\mathbf{M n}_{\mathbf{2}}^{\text {II }}\right\}$ fraction with elevating temperature, in contrast to the pure $\left\{\mathbf{M n}_{\mathbf{2}}^{\text {III }}\right\}$ plateau for compound $\mathbf{1}$ at the same temperature. Noticeable, the initial magnetic behavior is also recovered after decompression (Fig. S2, ESI†).

Magnetization as a function of applied magnetic field of compounds 1 and 2 were also measured at $2 \mathrm{~K}$ and at variable pressure (Fig. S3 and S4, ESI $\dagger$ ). $M$ vs. $H$ curves show a slight increase of starting steepness when applying pressure for each compound. Saturation values of magnetization are higher than

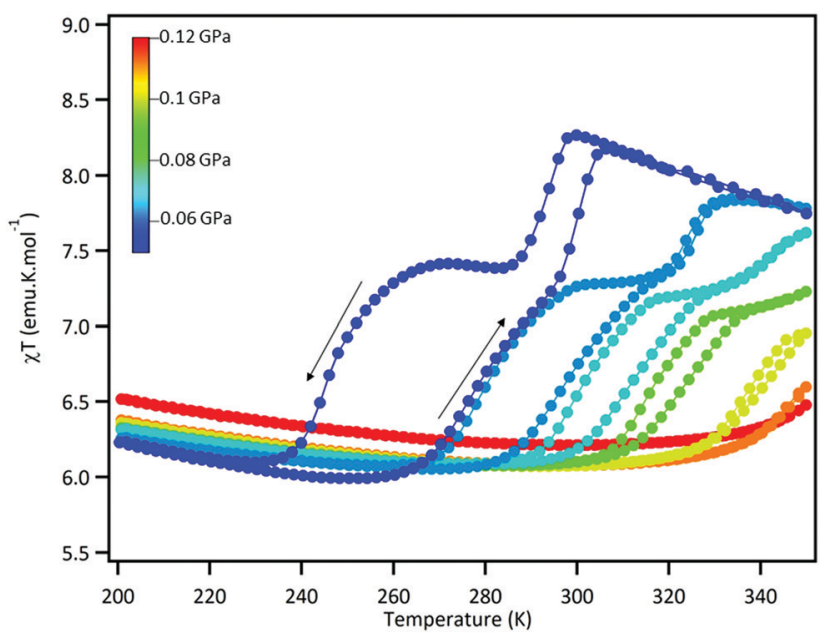

Fig. $2 \chi T$ vs. $T$ curves of compound 2 in the temperature range $200-350 \mathrm{~K}$ and at variable pressure ranging in between $10^{-4} \mathrm{GPa}$ (dark blue) and $0.12 \mathrm{GPa}$ (red). Black arrows indicate the direction of the hysteresis and solid lines only join the data points. 
for regular magnetic measurements using simple gelatin capsules, but they are still inferior to the theoretical value of $7 \mu_{\mathrm{B}}$ considering antiferromagnetic interactions between $2 \mathrm{Mn}$ (III) ions $(S=2)$ and one free radical $(S=1 / 2) \cdot{ }^{16,17}$ The spin-canting between the $2 \mathrm{D}$-layers is likely affected by pressure leading to higher magnetization saturation. Pressure appears to enhance magnetic interactions, as already suggested for a molecularbased magnet previously studied. ${ }^{10}$ The compression of the solids may induce a decrease of the distance between paramagnetic centers, increasing magnetic interaction strength.

These results show that the magnetic behaviors and redox forms of compounds $\mathbf{1}$ and $\mathbf{2}$ are hypersensitive to small changes in external applied pressure and far more than other VT compounds. ${ }^{8-11}$ The cell volume of compounds 1 and 2 has been determined by X-ray analyses at variable temperature in our previous work. ${ }^{16,17}$ They are equal to 2037 and $2047 \AA^{3}$ for the high temperature redox form and to 1974 and $1944 \AA^{3}$ for the low temperature redox form for $\mathbf{1}$ and $\mathbf{2}$ respectively. Compounds $\mathbf{1}_{\mathbf{L T}}$ and $\mathbf{2}_{\mathbf{L T}}$ redox forms $\left\{\mathbf{M n}_{\mathbf{2}}^{\text {III }}\right\}$ have a smaller cell volume than the $\mathbf{1}_{\mathbf{H T}}$ and $\mathbf{2}_{\mathbf{H T}}$ ones $\left\{\mathbf{M n}_{\mathbf{2}}^{\text {II }}\right\}$, mainly caused by the Jahn-Teller distortion of $\mathrm{Mn}$ (III) ions which induces a contraction of the lattice and a shortening of four out of six M-L bonds. Applying pressure strains the compound to contract its lattice and thus to move toward a smaller volume state to minimize its energy. This transfer is made possible thanks to the valence tautomeric conversion favored by the pressure. For $P \sim 0.1 \mathrm{GPa}$, the valence tautomeric conversion is happening at room temperature for both compounds. Under higher pressure, the LT redox form is stabilized on the all temperatures range below room temperature. This is what is observed for compound 3 in ambient-pressure; this seems to agree that $\mathrm{PF}_{6}{ }^{-}$ counter-anions induce a higher chemical pressure on the 2D coordination polymers than $\mathrm{ClO}_{4}^{-}$(1) or $\mathrm{BF}_{4}^{-}$(2), stabilizing the $\mathbf{3}_{\mathbf{L T}}$ tautomer under $330 \mathrm{K.}{ }^{17}$ Indeed, chemical and external pressures are different but in these compounds they should both mainly affect the counter-anion layers.

The evolution of the hysteresis shapes of compound 2 when applying pressure is particularly interesting. The first-step $\left(\mathbf{2}_{\mathbf{H T}} \Leftrightarrow \mathbf{2}_{\mathrm{INT}}\right)$ of the conversion becomes more gradual and is getting closer to the behavior observed for compound $3^{17}$ which has a twostep VT conversion between 380-330 K with thin hysteresis and gradual slope. The second-step of the conversion of $2\left(\mathbf{2}_{\mathbf{I N T}} \Leftrightarrow \mathbf{2}_{\mathbf{L T}}\right)$ shows a more abrupt but asymmetric hysteresis as observed for 1 . The crystallographic study of compound 2 has shown that the two steps of the conversion are caused by the successive oxidation of the two independent manganese centers. ${ }^{17}$ The first oxidation induces an elongation of the $\mathrm{Mn}$ (III) coordination spheres outside of the 2D-layer, whereas the Jahn-Teller axis is localized within the coordination polymer for the second oxidation. Thus, a more gradual slope of the first step under pressure increase can be due to a greater difficulty of the manganese to distort outside of the plane. Although the external pressure is applied isotropically on the polycrystalline powder, it certainly has the greatest effect on the interlayer space, and reducing it. Rearrangement is more difficult, slowing down the propagation of the 1st electron transfer for compound 2. The incomplete conversion of compound $\mathbf{1}$ can probably be explained for the same reasons. Thus, when pressure increases, it seems that compound 2 behaves more like compound 3, as well as compound $\mathbf{1}$. The hysteresis vanishing under high pressure can arise from a decrease of degree of freedom of the lamellar structure. A stronger rigidity of the structure may reduce the cooperativity. The asymmetric character of the hysteresis of $\mathbf{1}$ and the second hysteresis of 2 rises from a more gradual warming branch. When compounds are heating back, the crystal lattice has to decompress which is a disfavor process under high pressure.

Raman spectroscopy studies were previously carried out at ambient-pressure and variable temperature to follow the valence tautomeric conversion in compounds 1 and $2^{16,17}$ The formation of a new band close to $1617 \mathrm{~cm}^{-1}$ in their Raman spectra when decreasing temperature was found to evidence the VT conversion. This band arises from the reduction of the nitronyl nitroxide radical to its aminoxyl tautomer form. The band disappeared upon heating back, proving the reversible electron transfer. ${ }^{17}$ Based on these previous results, Raman measurements were done at room temperature under an excitation wavelength of $514 \mathrm{~nm}$ in a frequency range of $1000-1800 \mathrm{~cm}^{-1}$ at variable pressure $\left(10^{-4}-3.5 \mathrm{GPa}\right)$ for compound 1 (Fig. 3). The lowest trace (purple) is the ambient-pressure spectrum measured at room temperature, followed by the dark blue spectrum of the sample in the diamond anvil cell at only slightly higher pressure. The mechanical pressure variation was not fine enough to explore the hysteresis in Fig. 1 and 2 or to obtain another spectrum below the VT transition.

The Raman spectrum at atmospheric pressure shows a peak at $1564 \mathrm{~cm}^{-1}(\mathrm{~B})$, previously assigned to the $\nu(\mathrm{O}-\mathrm{N}=\mathrm{C})$ elongation of the nitronyl ring. ${ }^{17,23}$ As expected, this peak vanishes with increasing pressure and a new peak appears at $1600 \mathrm{~cm}^{-1}$ for $P>0.19 \mathrm{GPa}$. It broadens at higher pressures, particularly for $P>2.5 \mathrm{GPa}$. The peak around $1440 \mathrm{~cm}^{-1}(\mathrm{~A})$, assigned to $\nu(\mathrm{C}-\mathrm{N})$ of the imidazole,$^{23}$ is broadening with increasing pressure but does not show any shift, as this vibrational mode is not affected by the VT transition. Pressure is known to induce

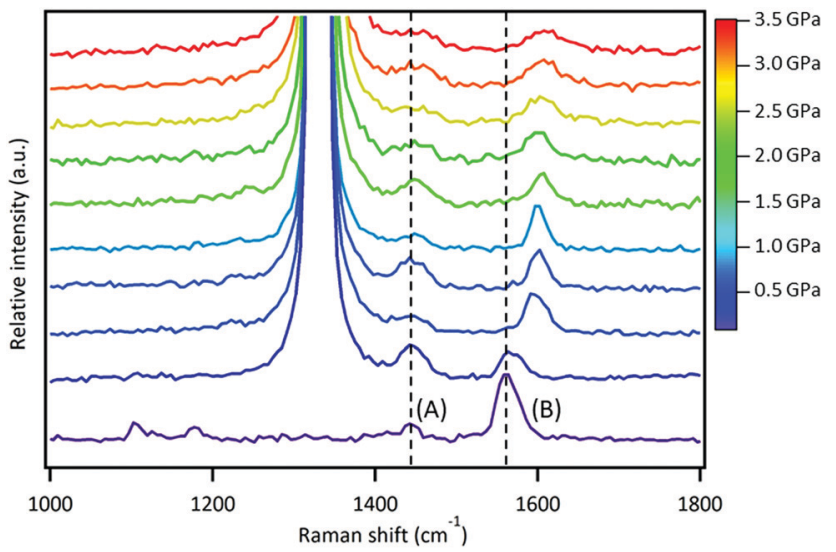

Fig. 3 Raman spectra of compound 1 at variable pressure measured at room temperature under an excitation wavelength of $514 \mathrm{~nm}$. The most intense peak at $1330 \mathrm{~cm}^{-1}$ in each spectrum corresponds to the characteristic Raman band of the diamond anvils. 
broadening and small shifts of Raman peaks caused by structure deformations. ${ }^{12}$ To confirm the disappearance/appearance switch of the peak in the $1564-1600 \mathrm{~cm}^{-1}$ region is due to the VT conversion and not only to general pressure effects, the maxima of the frequency band $\nu_{\max }$ for each of the two peaks (A and B) were plotted as a function of external applied pressure (Fig. S5, ESI $\dagger$ ). As can be seen, the plot for peak A shows small variations while for the peak in region $B$ there is a break with an abrupt shift to higher frequencies. After decompression all the peaks return to their initial position and shape (Fig. S6, ESI $\dagger$ ). As for Raman spectroscopy study at variable temperature, ${ }^{16,17}$ the appearance of a new Raman band at $+36 \mathrm{~cm}^{-1}$ from the $1564 \mathrm{~cm}^{-1}$ peak evidences the valence tautomeric conversion.

In conclusion, variable-pressure magnetic measurements and Raman spectra demonstrate how easy the VT conversion of these compounds, with manganese-nitronyl nitroxide radical 2D-frameworks, can be isothermally tuned by quite small increase of the pressure. This high sensitivity to pressure has a considerable impact on the shape of hysteresis and on the VT process. The electron transfers originate from two crystallographically independent populations of manganese ions and are impacted differently by compression, so that they occur in two successive steps that become more and more distinct as the pressure increases with narrowing of the hysteresis. The reason may be that the VT first step $\left\{\mathbf{M n}_{\mathbf{2}}^{\text {II }}\right\} \Leftrightarrow\left\{\mathbf{M n}^{\text {II }} \mathbf{M n}{ }^{\text {III }}\right\}$ leads to an elongation of the manganese coordination sphere outside of the coordination polymer plane and this is disfavored by compression. Whereas the VT second step $\left\{\mathbf{M n}^{\text {II }} \mathbf{M n}^{\text {III }}\right\} \Leftrightarrow\left\{\mathbf{M n}_{\mathbf{2}}^{\text {III }}\right\}$ leads to a distortion within the sheet and this seems to be easier under pressure. This is well illustrated by compound 2 whose magnetic behavior becomes increasingly comparable to that of compound 3 with pressure increase. These results are potentially interesting for technological applications in the field of temperature or pressure memory sensors and integration of these compounds in materials can be envision, in particular in nano-sized matrices taking into account the matrix effect highlighted after simple confinement.

This work was supported Université Claude Bernard Lyon 1, Centre National de la Recherche Scientifique (CNRS), Natural Sciences and Engineering Research Council (Canada) and JSPS.

\section{Conflicts of interest}

There are no conflicts to declare.

\section{Notes and references}

1 M. Feng, Z.-Y. Ruan, Y.-C. Chen and M.-L. Tong, Chem. Commun., 2020, 56, 13702.

2 A. Hauser and C. Reber, in 50 Years of Structure and Bonding - The Anniversary Volume, ed. D. M. P. Mingos, Springer International Publishing, Cham, 2017, pp. 291-312.

3 O. Sato, Nat. Chem., 2016, 8, 644-656.

4 C. Boskovic, in Spin-Crossover Materials: Properties and Applications, ed. M. A. Halcrow, John Wiley \& Sons, Ltd, 1st edn, 2013.

5 M. A. Halcrow, Spin-Crossover Materials: Properties and Applications, Wiley, 2013.

6 G. Poneti, L. Poggini, M. Mannini, B. Cortigiani, L. Sorace, E. Otero, P. Sainctavit, A. Magnani, R. Sessoli and A. Dei, Chem. Sci., 2015, 6, 2268-2274.

7 A. Rajput, A. K. Sharma, S. K. Barman, A. Saha and R. Mukherjee, Coord. Chem. Rev., 2020, 414, 213240.

8 C. Roux, D. M. Adams, J. P. Itie, A. Polian, D. N. Hendrickson and M. Verdaguer, Inorg. Chem., 1996, 35, 2846-2852.

9 A. Caneschi, A. Dei, F. F. de Biani, P. Gutlich, V. Ksenofontov, G. Levchenko, A. Hoefer and F. Renz, Chem. - Eur. J., 2001, 7, 3926-3930.

10 V. Ksenofontov, A. B. Gaspar and P. Gutlich, in Spin Crossover in Transition Metal Compounds III, ed. P. Gutlich and H. A. Goodwin, 2004, vol. 235, pp. 23-64.

11 B. Li, F. L. Yang, J. Tao, O. Sato, R. B. Huang and L. S. Zheng, Chem. Commun., 2008, 6019-6021.

12 Y. Suffren, F.-G. Rollet and C. Reber, Comments Inorg. Chem., 2011, 32, 246-276.

13 O. Reu, S. Ostrovsky, S. Decurtins, S. X. Liu and S. Klokishner, Eur. J. Inorg. Chem., 2017, 5356-5365.

14 J. A. DeGayner, K. Y. Wang and T. D. Harris, J. Am. Chem. Soc., 2018, 140, 6550-6553.

15 C. Fleming, D. Chung, S. Ponce, D. J. R. Brook, J. DaRos, R. Das, A. Ozarowski and S. A. Stoian, Chem. Commun., 2020, 56, 4400-4403.

16 A. Lannes, Y. Suffren, J. B. Tommasino, R. Chiriac, F. Toche, L. Khrouz, F. Molton, C. Duboc, I. Kieffer, J. L. Hazemann, C. Reber, A. Hauser and D. Luneau, J. Am. Chem. Soc., 2016, 138, 16493-16501.

17 C. Lecourt, Y. Izumi, L. Khrouz, F. Toche, R. Chiriac, N. BélangerDesmarais, C. Reber, O. Fabelo, K. Inoue, C. Desroches and D. Luneau, Dalton Trans., 2020, 49, 15646-15662.

18 G. G. Levchenko, V. Ksenofontov, A. V. Stupakov, H. Spiering, Y. Garcia and P. Gutlich, Chem. Phys., 2002, 277, 125-129.

19 K. Yokogawa, K. Murata, H. Yoshino and S. Aoyama, Jpn. J. Appl. Phys., 2007, 46, 3636-3639.

20 K. Y. Maryunina, X. Zhang, S. Nishihara, K. Inoue, V. A. Morozov, G. V. Romanenko and V. I. Ovcharenko, J. Mater. Chem. C, 2015, 3, 7788-7791.

21 N. A. Artiukhova, G. V. Romanenko, A. S. Bogomyakov, I. Y. Barskaya, S. L. Veber, M. V. Fedin, K. Y. Maryunina, K. Inoue and V. I. Ovcharenko, J. Mater. Chem. C, 2016, 4, 11157-11163.

22 Y. Raza, F. Volatron, S. Moldovan, O. Ersen, V. Huc, C. Martini, F. Brisset, A. Gloter, O. Stéphan, A. Bousseksou, L. Catala and T. Mallah, Chem. Commun., 2011, 47, 11501-11503.

23 C. Lecourt, W. Madanamoothoo, V. Ferreol, N. Belanger-Desmarais, L. Khrouz, J. B. Tommasino, C. Reber, C. Desroches and D. Luneau, Dalton Trans., 2019, 48, 13378-13387. 